\title{
The Relation of Serum 25-Hydroxy Vitamin D3 with Inflammation and Indirect Markers of Thrombotic Activity in Patients with End Stage Renal Disease
}

\section{Bennur Esen ${ }^{1 *}$, Ahmet Engin Atay ${ }^{1}$, Irfan Sahin², Emel Saglam Gokmen ${ }^{1}$, Suat Hayri Kucuk ${ }^{3}$, Sefik Eser Ozyurek ${ }^{4}$, Ozlem Kaptanogullari ${ }^{5}$ and Numan Gorgulu ${ }^{1}$}

${ }^{1}$ Bagcilar Education and Research Hospital, Internal Medicine and Nephrology, Istanbul, Turkey

${ }^{2}$ Bagcilar Education and Research Hospital, Cardiology, Istanbul, Turkey

${ }^{3}$ Bagcilar Education and Research Hospital, Biochemistry, Istanbul, Turkey

${ }^{4}$ Bagcilar Education and Research Hospital, Department of Obstetrics and Gynecology, Istanbul, Turkey

${ }^{5}$ Bakırkoy Dr. S. Konuk Education and Research Hospital, Internal Medicine and Nephrology, Istanbul, Turkey

${ }^{*}$ Corresponding author: Bennur Esen, Division of Nephrology, Department of Internal Medicine, Bagcilar Research and Training Hospital, Istanbul, Turkey, E-mail: bennuresen@yahoo.com

Received date: March 31, 2016; Accepted date: April 29, 2016; Published date: May 02, 2016

Citation: Esen B, Atay AE, Sahin I, Gokmen ES, Kucuk SH, et al. (2016) The Relation of Serum 25-Hydroxy Vitamin D3 with Inflammation and Indirect Markers of Thrombotic Activity in Patients with End Stage Renal Disease. J Clin Exp Nephrol 1: 8. DOI: 10.21767/2472-5056.100008

Copyright: (C) 2016 Esen B, et al. This is an open-access article distributed under the terms of the Creative Commons Attribution License, which permits unrestricted use, distribution, and reproduction in any medium, provided the original author and source are credited.

\section{Abstract}

Background: Patients with end stage renal disease (ESRD) have increased inflammatory and thrombotic activity. Lacks of available data exist on pro-inflammatory and prothrombotic properties of low serum 25-OH-D3 level. We aimed to analyse the association of $25-\mathrm{OH}$ vitamin $\mathrm{D}$ with indirect markers of inflammatory and thrombotic activity in patients on haemodialysis (HD) or peritoneal dialysis (PD)

Methods: A total of 104 patients with ESRD receiving renal replacement therapy (RRT) were enrolled into this prospective study. Seventy patients on HD and 34 age matched patients on PD with similar duration of ESRD and RRT were from same geographical area and had similar sunlight exposure. The mean age of patients on HD and PD were $56.59 \pm 18.19$ years and $53.26 \pm 10.6$ years; respectively. Fasting blood samples were obtained before dialysis session to analyse serum creatinine, C-reactive protein (CRP), erythrocyte sedimentation rate (ESR), leukocyte count, platelet count, platecrit (PCT), haemoglobin, haematocrit and 25-OH-D3.

Results: There was no significant difference between patients on PD and HD in terms of serum calcium, phosphorus, parathormon, CRP, ESR, ferritin and bicarbonate levels. Patients on PD had significantly lower level of $25-\mathrm{OH}-\mathrm{D3}$ (9.35 \pm 7.69 vs $4.71 \pm 3.01$, p: 0.0001 ). Leukocyte count, platelet count, haemoglobin, haematocrit, RDW, neutrophil ratio, monocyte ratio, neutrophil count and PCT were significantly higher in PD patients ( $p: 0.001$, $p<0.001, p<0.001, p: 0.002, p: 0.020, p: 0.034, p: 0.009, p:$ 0.001 , p: 0.001 ; respectively). There was a significant association between 25 hydroxy vitamin D3 with, CRP, ESR and haematocrit in PD group but not in HD group ( $p: 0.019$, $\mathrm{p}: 0.002$, p:0.025 respectively). Female patients in both groups had lower 25-OH-D3 level than male dialysis patients (p: 0.0001). Multiple regression analysis revealed out a significant effect of CRP, sedimentation, haematocrit, platelet count and PCT on D vitamin level ( $p: 0.022$ ).

Conclusion: Low serum 25-OH-D3 level reflects increased inflammatory and platelet activity in PD patients.

Keywords: 25-hydroxy vitamin D; Inflammation; Thrombotic activity; Renal replacement; Dialysis

\section{Introduction}

Hydroxyvitamin D3 (25-OH-VitD3) is the inactive form of vitamin $D$ that regulates calcium $(\mathrm{Ca})$ and phosphate $(\mathrm{P})$ homeostasis [1]. The role of vitamin D on $\mathrm{Ca}, \mathrm{P}$ and parathormon (PTH) metabolism, development of neuronal cells and maturation of immune cells are well-known. Vitamin D deficiency is a risk factor for infectious, autoimmune, neurodegenerative and cardiovascular disorders (CVD) as well as frequently seen in patients with diabetes, osteoporosis, and cancer [2]. Deficiency of vitamin D is frequently observed in patients with end-stage renal disease (ESRD) undergoing renal replacement therapies (RRT) [3]. Ciccione et al. determined that vitamin $\mathrm{D}$ deficiency is associated with cardiovascular mortality as a result of heart failure, myocardial infarction and sudden cardiac death [4].

Chronic kidney disease (CKD) is characterized by an increase in oxidative stress and inflammation [5]. Patients on RRT have predisposition to coagulation which is shown by increased procoagulant factors (fibrinogen, von Willebrand factor) and activated coagulation markers (D-Dimer and prothrombin fragments), decreased endogenous anticoagulants (antithrombin, protein $\mathrm{C}$ and S) and fibrinolytic activity [6]. Cardiovascular diseases in patients without traditional CVD risk 
factors suggest the role of non-traditional risk factors or pathogenetic mechanisms including inflammation, oxidative stress and hormonal alterations. Although there are controversial reports, low grade inflammation and low serum 25-OH-VitD3 level are recently proposed risk factors [7]. Epidemiologic studies showed that vitamin D has potential antiinflammatory effect through regulation of inflammatory mediators and an independent association with elevated CRP and decreased kidney function [8].

Platelets play important role in inflammatory process as well as thrombosis. Plateletcrit (PCT) and platelet distribution width (PDW) are platelet parameters reflecting thrombotic activity [9]. PCT is a parameter of platelet concentration and has potential to reflect changes in the rate of platelet production and activity [10]. PCT is also related with thrombotic activity that predicts cardiovascular mortality in ESRD. Some researchers have demonstrated its potential to predict thrombosis risk [11]. Also, PCT value is a beneficial parameter to determine severity of infection [11].

In the present study, we aimed to analyse the relation of vitamin $\mathrm{D}$ with parameters inflammation and platelet reactivity in patients with ESRD receiving RRT.

\section{Material and Methods}

Between January and March 2015, 104 patients with ESRD (70 patients on haemodialysis (HD) (31F/39M) and 34 age matched patients on peritoneal dialysis (PD) $(22 \mathrm{~F} / 12 \mathrm{M})$ ) receiving $\mathrm{RRT}$ and similar sunlight exposure were enrolled into this prospective study. Patients on PD and HD were advised to expose sunlight at least 1 hour/day. Duration of sunlight exposure was determined by interviewing with the patients. The study population was consisting of Turkish citizens from same ethnic background. Study was carried out in the same region of Istanbul between January and March 2015. Patients with active immunologic or tumoral disease, immobility, obesity, smoking, severe anaemia (haematocrit<27\%), acute inflammatory or infectious episodes in last 3 months, connective tissue disorder, active treatment with immunotherapy or immunosuppressive medications were excluded.

Patients on PD and HD were regularly followed up in Dialysis Center and Nephrology outpatient service of Bagcilar Education and Research Hospital. All participants were under dietary restrictions as recommended in the K-DOQI guideline, and were followed by a physician and a dietician [12]. Hemogram and serum electrolyte levels including potassium, sodium and calcium were analysed by monthly intervals. According to results, dietary recommendations were updated by same dietician and nephrologist. Entire patients underwent to physical examination, and demographic features of participants were recorded. Ethics committee of Bagcilar Education and Research Hospital approved the study. Written informed consent was obtained from all participants.

Blood samples were collected after 8-hour fasting period. Hemogram parameters were analysed after taken into tubes with EDTA using an automatic blood counter (XE-5000; Sysmex Corp, Kobe, Japan). Biochemical variables including serum $\mathrm{Ca}, \mathrm{P}$, albumin, low density lipoprotein (LDL) was analysed by photometric method in Siemens Advia 1800 device. Serum parathormon (PTH) level was analysed by electrochemiluminescence immunoassay (ECLIA) method in Cobas auto analyser (Roche Diagnostics $\mathrm{GmbH}$, Mannheim, Germany). C reactive protein (CRP) was analysed by photometric method in Roche Diagnostics Cobas 8000 analyser (Roche Diagnostics $\mathrm{GmbH}$, Mannheim, Germany). Erythrocyte sedimentation rate (ESR) was analysed by Westergren method in Ves-Matic Cube 200 (DIESSE Diagnostica Senese Milano, Italy).

Serum levels of $25 \mathrm{OH}-\mathrm{D} 3$ were examined in roche cobas 6000 immunoassay analyser by ECLIA method. According to K-DOQI, severe, moderate and mild 25-OH-D deficiencies were $<5 \mathrm{ng} / \mathrm{ml}$, $5-15 \mathrm{ng} / \mathrm{ml}$ and $15-29 \mathrm{ng} / \mathrm{ml}$ [12]. PTH levels were analysed in every 3 months, and vitamin $D$ was administered on the basis of achieving a PTH level $<300 \mathrm{IU} / \mathrm{L}$.

Dialysis adequacy was determined by measurement of kT/v. Patients on both groups have negligible residual renal function as seen by glomerular filtration rate of $<10 \mathrm{ml} /$ minute and urine output of $<100 \mathrm{ml} /$ day. None have a remarkable proteinuria or body mass index $(\mathrm{BMI})>30 \mathrm{~kg} / \mathrm{m}^{2}$. Serum bicarbonate $\left(\mathrm{HCO}_{3}\right)$ level was assessed using blood gas analysis. Serum iron and iron binding capacity were determined by chemiluminescent enzyme Immunoassay with deproteinized ferrozinc method. Ferritin levels were analysed by immunoturbidimetric test method.

\section{Statistical analysis}

SPSS 22.0 (SPSS, Chicago, Illinois, USA) package programme was used for statistical analysis. Data were expressed as mean \pm SD. Shapiro Wilks test was used to analyse distribution of the variables. Parametric variables were compared with independent $t$ test, ordinal data were compared with Mann Whitney $U$ test, and non-parametric variables were compared with chi-square test. Quantitative parameters were compared with Kruskal Wallis test. Spearman's rho correlation test was used to evaluate the relation of parametric variables. A $p$ value $<0.05$ was considered significant.

\section{Result}

There were 70 patients $(31 \mathrm{~F} / 39 \mathrm{M})$ on $\mathrm{HD}$ and 34 age matched patients (22F/12M) on PD. The mean age ( $56.59 \pm 18.19$ vs 53.26 \pm 10.6 years) and RRT duration $(41.1 \pm 42.52$ vs $29.09 \pm 20.37$ months) of HD and PD were similar. Entire participants were non obese $\left(\mathrm{BMl}<30 \mathrm{~kg} / \mathrm{m}^{2}\right)$. All the patients in both groups survived during the study period. Patients on PD had significantly lower level of $25-\mathrm{OH}-\mathrm{D} 3(9.35 \pm 7.69$ vs $4.71 \pm 3.01 ; \mathrm{p}$ : 0.0001$)$. There was no significant difference between patients on $P D$ and $H D$ in terms of serum Ca, P, CaxP, PTH, albumin, iron, CRP, ESR, ferritin and bicarbonate levels. Leukocyte count $(9.33 \pm 2.92$ vs $7.54 \pm$ 2.31), platelet count $(269.53 \pm 82.47$ vs $211.17 \pm 70.34)$, haemoglobin (11.69 \pm 1.4 vs $10.44 \pm 1.67)$, haematocrit (36.06 \pm 5.18 vs $32.24 \pm 5.97)$, neutrophil ratio (66.96 \pm 9.13 vs $62 ., 92 \pm$ $8.62)$ and PCT $(0.19 \pm 0.06$ vs $0.15 \pm 0.05)$ were significantly 
higher in PD patients ( $p: 0.001, p<0.001, p<0.001, p: 0.002, p$ : 0.034 and $\mathrm{p}: 0.001$; respectively) (Table 1 ).

Table 1: Comparison of variables between patients on haemodialysis and peritoneal dialysis. RDW: Red Cell Distribution Width; MCV: Mean Corpuscular Volume; MPV: Mean Platelet Volume; PCT: Platecrite; PDW: Platelet Distribution Width; RRT: Renal Replacement Therapy; PTH: Parathormon; CRP: C-Reactive Protein.

\begin{tabular}{|c|c|c|c|}
\hline & Haemodialysis & Peritoneal dialysis & $\mathbf{p}$ \\
\hline 25-OH-Vitamin D & $9.35 \pm 7.69$ & $4.71 \pm 3.01$ & $<0.001$ \\
\hline Age & $56.59 \pm 18.19$ & $53.26 \pm 10.66$ & 0.241 \\
\hline Duration of RRT & $42.1 \pm 42.52$ & $29.09 \pm 20.37$ & 0.569 \\
\hline KT.V & $1.45 \pm 0.18$ & $2.34 \pm 0.37$ & $<0.001$ \\
\hline Calcium (Ca) & $8.71 \pm 0.93$ & $8.72 \pm 1.07$ & 0.964 \\
\hline Phosphorus (P) & $5.05 \pm 1.67$ & $4.83 \pm 1.26$ & 0.509 \\
\hline CaXP & $43.64 \pm 15.25$ & $41.88 \pm 10.98$ & 0.558 \\
\hline PTH & $522.53 \pm 751.41$ & $386.41 \pm 379.78$ & 0.694 \\
\hline CRP & $12.87 \pm 14.51$ & $18.19 \pm 30.87$ & 0.588 \\
\hline Total protein & $6.76 \pm 0.57$ & $6.76 \pm 0.55$ & 0.94 \\
\hline Albumin & $3.87 \pm 0.46$ & $3.74 \pm 0.44$ & 0.177 \\
\hline Iron & $60.36 \pm 30.75$ & $67.56 \pm 28.99$ & 0.267 \\
\hline $\begin{array}{l}\text { Iron binding } \\
\text { capacity }\end{array}$ & $238.32 \pm 51.25$ & $246.03 \pm 51.29$ & 0.483 \\
\hline Ferritin & $564.33 \pm 382.8$ & $455.89 \pm 334.56$ & 0.171 \\
\hline Sedimentation & $51.34 \pm 27.66$ & $52.69 \pm 19.28$ & 0.782 \\
\hline Bicarbonate & $20.92 \pm 2.78$ & $22.17 \pm 3.66$ & 0.061 \\
\hline Leukocyte count & $7.54 \pm 2.31$ & $9.33 \pm 2.92$ & 0.001 \\
\hline Haemoglobin & $10.44 \pm 1.67$ & $11.69 \pm 1.4$ & $<0.001$ \\
\hline Hematocryte & $32.24 \pm 5.97$ & $36.06 \pm 5.18$ & 0.002 \\
\hline Platelet & $211.17 \pm 70.34$ & $269.53 \pm 82.47$ & $<0.001$ \\
\hline RDW & $14.26 \pm 1.69$ & $13.44 \pm 1.43$ & 0.02 \\
\hline MCV & $89.9 \pm 5.46$ & $87.99 \pm 5.17$ & 0.099 \\
\hline MPV & $7.33 \pm 1.45$ & $7.12 \pm 0.97$ & 0.382 \\
\hline РСT & $0.15 \pm 0.05$ & $0.19 \pm 0.06$ & 0.001 \\
\hline PDW & $18.23 \pm 2.39$ & $17.89 \pm 1.87$ & 0.485 \\
\hline Lymphocyte ratio & $25.27 \pm 9.78$ & $22.4 \pm 7.49$ & 0.144 \\
\hline $\begin{array}{l}\text { Lymphocyte } \\
\text { count }\end{array}$ & $1.8 \pm 0.67$ & $1.99 \pm 0.74$ & 0.19 \\
\hline Monocyte ratio & $7.92 \pm 2.58$ & $6.82 \pm 1.53$ & 0.009 \\
\hline Monocyte count & $0.59 \pm 0.24$ & $0.63 \pm 0.21$ & 0.453 \\
\hline Neutrophil ratio & $62.92 \pm 8.62$ & $66.96 \pm 9.13$ & 0.034 \\
\hline
\end{tabular}

\begin{tabular}{|l|l|l|l} 
Neutrophil count & $4.81 \pm 1.81$ & $6.38 \pm 2.56$ & 0.001
\end{tabular}

When all patients were considered, correlation analysis indicated a significant association between 25-OH-D3 with age, platelet count, PCT, haematocrit, bicarbonate, albumin, ESR, monocyte ratio and neutrophil count. (r:-0.214, p:0.035; r:-0.266, p:0.009; r:-0.303, p:0.003; r:0.219, p:0.033; r:-0.255, p: 0.013 ; r:0.299, p:0.003; r:-0.308, p:0.003; r:0.238, p:0.020; $r:-0.215, \mathrm{p}: 0.036$; respectively) (Table 2). Female patients in both groups had lower $25-\mathrm{OH}-\mathrm{D} 3$ level than male dialysis patients ( $p$ : 0.0001).

Table 2: The relation of variables with $25-\mathrm{OH}-\mathrm{Vitamin} \mathrm{D} 3$ in both groups. MCV: Mean Corpuscular Volume; PCT: Platecrite.

\begin{tabular}{|l|l|l|}
\hline & $\mathbf{r}$ & $\mathbf{p}$ \\
\hline Sedimentation & -0.308 & 0.003 \\
\hline Bicarbonate & -0.255 & 0.013 \\
\hline Hematocryte & 0.219 & 0.033 \\
\hline Platelet & -0.266 & 0.009 \\
\hline MCV & 0.276 & 0.007 \\
\hline PCT & -0.303 & 0.003 \\
\hline Monocyte ratio & 0.238 & 0.02 \\
\hline Neutrophil count & -0.215 & 0.036 \\
\hline Age & -0.214 & 0.035 \\
\hline Albumin & 0.299 & 0.003 \\
\hline
\end{tabular}

There was a significant association between 25 hydroxy vitamin D3 with, CRP, ESR and haematocrit in PD group ( $r$ : -0.419 , p: 0.019 ; $r:-0.534$, p: $0.002 ; r: 0,401, p: 0.025$ respectively) (Table 3 ).

Table 3: Variables significantly related to $25-\mathrm{OH}-$ Vitamin $D$ in peritoneal dialysis group. CRP: C-Reactive Protein.

\begin{tabular}{|l|l|l|}
\hline & $\mathbf{r}$ & $\mathbf{p}$ \\
\hline CRP & -0.419 & 0.019 \\
\hline Sedimentation & -0.534 & 0.002 \\
\hline Hematocryte & 0.401 & 0.025 \\
\hline
\end{tabular}

Backward regression analysis revealed out a significant effect of CRP, sedimentation, haematocrit, platelet count and platecrit on D vitamin level in PD group ( $p: 0.022 ; p<0.05)$. In the model, the most significant variables in relation with vitamin $D$ were sedimentation and platecrite ( $p: 0.007$ and $p: 0.019$ respectively) (Table 4).

According to post hoc analysis, the power of the study was $99.3 \%$

Table 4: Multiple regression analysis examining the effect of variables on vitamin D in PD group. 


\begin{tabular}{|l|l|l|l|l|l|l|l|}
\hline & & & & & & $95 \%$ Cl \\
\hline & B & S.E. & Beta & $\mathbf{t}$ & $\mathbf{p}$ & Lower bound & Upper bound \\
\hline PD 3 (Constant) & 10.908 & 2.319 & & 4.704 & 0.001 & 6.141 & 15.676 \\
\hline Sedimentation & -0.072 & 0.025 & -0.513 & -2.909 & 0.007 & -0.123 & -0.021 \\
\hline PCT & -40.400 & 16.192 & -0.799 & -2.495 & 0.019 & -73.684 & -7.117 \\
\hline
\end{tabular}

\section{Discussion}

To the best of our knowledge, this is the first report that examined the relation of $25-\mathrm{OH}-\mathrm{Vit}$ D3 with inflammation parameters and indirect markers of platelet activity by comparing patients on HD and PD. The present study indicated that PD patients have lower vitamin D status than HD patients. Metabolic parameters were better controlled in PD patients however inflammatory and thrombotic activities are also increased in PD group.

In general population, a strong relationship between low 25$\mathrm{OH}-\mathrm{D} 3$ and high CRP levels was determined [13]. In experimental animal studies, it has been shown that long-term vitamin $D$ deficiency has potential to provoke chronic inflammation [14]. Activation of vitamin $D$ by kidney regulates transcriptions of inflammatory cytokines and prevents excessive production of inflammatory cells [14]. Vitamin D has stimulatory effect on antiinflammatory cytokines like IL-4, IL-5 and IL-10, and inhibitory effect on pro-inflammatory cytokines including IL-2, IL-3 and TNF-alpha. Also vitamin D modulates conversion of CD4 T cells to regulatory cells [15]. Although CRP is an important predictor for mortality in HD patients, its role in predicting outcome in PD patients is unclear [16].

Disease and RRT duration have significant impact on inflammatory markers [17]. As duration of dialysis therapy increases, decline of residual renal function, deterioration in volume-nutritional status, and augmentation in atherosclerotic complications become evident that all are associated with activated immune system [16]. Our patients on PD and HD have similar and adequate $\mathrm{kT} / \mathrm{v}$ value that helped to eliminate the impact of dialysis adequacy on serum 25-OH-Vit D3.

Peritoneal cells exposed to dialysis fluid with high glucose content which activates leukocyte infiltration and inflammatory cytokine production in PD patients [18]. Peritoneal mesothelial cells express components of RAAS which is upregulated in acute inflammation suggesting the link between inflammation and fluid-electrolyte balance [19]. Also uraemia may provoke inflammation in peritoneum of HD patients without a history of PD which pointed out the importance of dialysis adequacy [20].

Neutrophils are white blood cells participated in inflammatory process [7]. Previously, neutrophil/ lymphocyte (N/L) ratio was reported as an inflammatory marker that had significant correlation with other inflammatory markers [21]. HD and PD procedures stimulate complement cascade. Platelet-neutrophil micro aggregates and activation of neutrophils are related with exacerbation of complement activation [22,23]. In accordance with literature, $\mathrm{N} / \mathrm{L}$ ratio was correlated with inflammatory markers in the present study.
Patients with chronic inflammation are more frequently become anaemic as a cause of inhibitory effect of inflammatory mediators on iron supply to bone marrow. In a study on 16.301 patients with CKD, Kendrick et al determined an association between low 25-OH-D3 and high CRP with anaemia [24]. In our study, correlation of low serum 25-OH-D3 with anaemia is considered as an evidence of suppressive role of inflammation on bone marrow function.

In addition to increased inflammatory activity in patients with low serum 25-OH-VitD3, recently it has been proved that individuals with hypovitaminosis $D$ are tend to experience more frequent thrombotic events [25]. Platelet activation plays central role in the pathogenesis of haemostasis and thrombosis [26]. Ugur et al. showed a relation between platelet count (PLT) and platecrit (PCT) which reflect platelet activation and potential thrombotic event risk [27]. Platecrit predict the risk of thrombotic events and is one of fibrotic factors related with cardiovascular mortality in ESRD patients [26]. Fu et al. showed that platelet markers are beneficial to monitorize thrombosis related acute rejection risk in renal transplantation [28]. Correa et al. observed an inverse relation between decreased 25-OH-D3 level with platelet count and PCT [29]. We determined decreased level of 25-OH-D3 and increased platelet count and PCT which suggesting inflammatory and thrombotic role of decreased 25-OH-D3 level in patients receiving RRT.

Older age, obesity, vegetarian diet, living in a region with low UV radiation and avoiding sun exposure are common risk factors associated with decreased vitamin D production or uptake [30]. In most of the populations, females have twice the risk of vitamin D deficiency [30]. Because adipose tissue is a source of subclinical inflammation, and adipocytes express receptors for inflammatory mediators, we included non-obese dialysis patients with similar BMI and body-fat composition [31]. On the other hand, because serum $250 \mathrm{H}-\mathrm{D}$ levels exhibit seasonal and regional oscillations, the study performed in the same session and region [29]. Smoking may interact with inflammation and thrombosis. Also, smoking was an exclusion criteria for our study.

The present study has some potential drawbacks. First, relatively low sample size was the major limitation of the study. A possible explanation of low sample size is strict exclusion criterias such as active immunologic or tumoral disease, immobility, obesity, smoking, severe anaemia (haematocrit $<27 \%$ ), acute inflammatory or infectious episodes in last 3 months, connective tissue disorder, active treatment with immunotherapy or immunosuppressive medications. Second, single point measurement of PCT and 25 hydroxy vitamin D3 limits the significance of the results. Third, the relation of low 
vitamin $D$ with radiologic and angiographic signs of atherosclerosis may help for better understanding of exact relationship.

In conclusion, we demonstrated thrombotic and inflammatory activity of decreased $25 \mathrm{OH}$ vitamin D3 level in PD patients. Further studies with large sample size are warranted to reach more precise conclusions.

\section{Compliance with Ethical Standards}

Funding: This study was funded by authors of the manuscript.

Conflict of interest: On behalf all authors, the corresponding author state that there is no conflict of interest with regard to this manuscript.

Ethical approval: All procedures performed in studies involving human participants were in accordance with the ethical standards of the institutional and/or national research committee and with the 1964 Helsinki declaration and its later amendments or comparable ethical standards.

Informed consent: Informed consent was obtained from all individual participants included in the study.

\section{References:}

1. DeLuca HF (2004) Overview of general physiologic features and functions of vitamin D. Am J Clin Nutr 80: 1689S-1696S.

2. Dusso AS, Brown AJ, Slatopolsky E (2005) Vitamin D. Am J Physiol Renal Physiol 289: F8-28.

3. Mehrotra A, Leung WY, Joson T (2015) Nutritional vitamin D supplementation and health-related outcomes in hemodialysis patients: a protocol for a systematic review and meta-analysis. Syst Rev 21: 4-13.

4. Ciccone MM, Zito A, Dentamaro I, Vestito D, Scicchitano P, et al. (2015) [Vitamin D deficiency and cardiovascular diseases]. G Ital Cardiol (Rome) 16: 16-20.

5. Tucker PS, Scanlan AT, Dalbo VJ (2015) Chronic kidney disease influences multiple systems: describing the relationship between oxidative stress, inflammation, kidney damage, and concomitant disease. Oxid Med Cell Longev 29: 3006-3015.

6. Dong J, Li YJ, Yang ZK, Xu R (2014) Prognostic value of serum von Willebrand factor, but not soluble ICAM and VCAM, for mortality and cardiovascular events is independent of residual renal function in peritoneal dialysis patients. Perit Dial Int 34: 706-713.

7. Donate-Correa J, Domínguez-Pimentel $\mathrm{V}$, Méndez-Pérez $\mathrm{ML}$, Muros-de-Fuentes M, Mora-Fernández C, et al. (2014) Selective vitamin $D$ receptor activation as anti-inflammatory target in chronic kidney disease. Mediators Inflamm 67: 475-478.

8. Lai S, Molfino A, Russo GE, Testorio M, Galani A, et al. (2015) Cardiac, Inflammatory and Metabolic Parameters: Hemodialysis versus Peritoneal Dialysis. Cardiorenal Med 5: 20-30.

9. Song YH, Park SH, Kim JE, Ahn JY, Seo YH, et al. (2009) [Evaluation of platelet indices for differential diagnosis of thrombocytosis by ADVIA 120]. Korean J Lab Med 29: 505-509.

10. Subramaniam N, Mundkur S, Kini P, Bhaskaranand N, Aroor S (2014) Clinicohematological study of thrombocytosis in children. ISRN Hematol 2014: 389257.
11. Mutlu H, Artis TA, Erden A, Akca Z (2013) Alteration in mean platelet volume and platicrit values in patients with cancer that developed thrombosis. Clin Appl Thromb Hemost 19: 331-333.

12. London G, Coyne D, Hruska K, Malluche HH, Martin KJ (2010) The new kidney disease: improving global outcomes (KDIGO) guidelines-expert clinical focus on bone and vascular calcification. Clin Nephrol 74: 423-432.

13. Dong J, Li YJ, Yang ZK, Xu R (2014) Prognostic value of serum von Willebrand factor, but not soluble ICAM and VCAM, for mortality and cardiovascular events is independent of residual renal function in peritoneal dialysis patients. Perit Dial Int 34: 706-713.

14. Kiani AN, Fang H, Magder LS, Petri M (2013) Vitamin D deficiency does not predict progression of coronary artery calcium, carotid intima-media thickness or high-sensitivity C-reactive protein in systemic lupus erythematosus. Rheumatology (Oxford) 52: 2071-2076.

15. Deluca HF, Cantorna MT (2001) Vitamin D: its role and uses in immunology. FASEB J 15: 2579-2585.

16. Zhu L, Kong M, Han YP, Bai L, Zhang X, et al. (2015) Spontaneous liver fibrosis induced by long term dietary vitamin $D$ deficiency in adult mice is related to chronic inflammation and enhanced apoptosis. Can J Physiol Pharmacol 93: 385-94.

17. Wang AY, Woo J, Lam CW, Wang M, Sea MM, et al. (2003) Is a single time point $\mathrm{C}$-reactive protein predictive of outcome in peritoneal dialysis patients? J Am Soc Nephrol 14: 1871-1879.

18. Bilen Y, Cankaya E, Bilen N, Keles M, Erdem F, et al. (2014) Peritonitis incidence was correlated with duration of peritoneal dialysis rather than leptin or neutrophil to lymphocyte $(\mathrm{n} / \mathrm{l})$ ratio in peritoneal dialysis patients. Eurasian J Med 46: 145-150.

19. Lai KN, Leung JC (2010) Inflammation in peritoneal dialysis. Nephron Clin Pract 116: c11-18.

20. Wang AY, Wang M, Woo J, Lam CW, Lui SF, et al. (2004) Inflammation, residual kidney function, and cardiac hypertrophy are interrelated and combine adversely to enhance mortality and cardiovascular death risk of peritoneal dialysis patients. J Am Soc Nephrol 15: 2186-2194.

21. Nessim SJ, Perl J, Bargman JM (2010) The renin-angiotensinaldosterone system in peritoneal dialysis: is what is good for the kidney also good for the peritoneum? Kidney Int 78: 23-28.

22. Inal S, Okyay GU, Ulu MS, Kıdır V, Altuntas A, et al. (2014) The effect of biocompatible peritoneal dialysis solutions on neutrophil to lymphocyte ratio. Ren Fail 36: 1239-1243.

23. Itoh S, Takeshita K, Susuki C, Shige-Eda K, Tsuji T (2008) Redistribution of P-selectin ligands on neutrophil cell membranes and the formation of platelet-neutrophil complex induced by hemodialysis membranes. Biomaterials 29: 3084-3090.

24. Kendrick J, Targher G, Smits G, Chonchol M (2009) 25Hydroxyvitamin $D$ deficiency and inflammation and their association with hemoglobin levels in chronic kidney disease. Am J Nephrol 30: 64-72.

25. Piantoni S, Andreoli L, Allegri F, Meroni PL, Tincani A (2012) Low levels of vitamin $D$ are common in primary antiphospholipid syndrome with thrombotic disease. Reumatismo 64: 307-313.

26. Li S, Zhu CG, Guo YL, Xu RX, Zhang Y, et al. (2015) The relationship between the plasma PCSK9 levels and platelet indices in patients with stable coronary artery disease. J Atheroscler Thromb 22: 76-84. 
27. Ugur M, Ayhan E, Bozbay M, Çiçek G, Ergelen M, et al. (2014) The independent association of plateletcrit with long-term outcomes in patients undergoing primary percutaneous coronary intervention. 2014 J Crit Care 29: 978-981.

28. Fu S, Liang Y, Yu L, Luo M, Wang Y, et al. (2015) [The correlation between platelet parameters and acute rejection after renal transplantation]. Nan Fang Yi Ke Da Xue Xue Bao 35: 413-416.

29. Correa P, Segersten U, Hellman P, Akerstrom G, Westin G (2002) Increased 25-hydroxyvitamin D3 1alpha-hydroxylase and reduced 25-hydroxyvitamin D3 24-hydroxylase expression in parathyroid tumors--new prospects for treatment of hyperparathyroidism with vitamin d. J Clin Endocrinol Metab 87: 5826-5829.

30. Lindqvist PG (2014) Cardiology Patient Page. On the possible link between vitamin $D$ deficiency and cardiovascular disease: should we D-lighten our lives? Circulation 129: e413-414.

31. Lai KN, Tang SC, Leung JC (2007) Mediators of inflammation and fibrosis. Perit Dial Int 27 Suppl 2: S65-71. 